\title{
Sound omission related brain responses in children
}

\author{
Tjerk T. Dercksen ${ }^{\text {a,b, }}$, Andreas Widmann ${ }^{\mathrm{a}, \mathrm{c}}$, Florian Scharf ${ }^{\mathrm{d}}$, Nicole Wetzel ${ }^{\mathrm{a}, \mathrm{b}, \mathrm{e}}$ \\ ${ }^{a}$ Leibniz Institute for Neurobiology, Brenneckestraße 6, 39118 Magdeburg, Germany \\ ${ }^{\mathrm{b}}$ Center for Behavioral Brain Sciences, Universitätsplatz 2, D-39106 Magdeburg, Germany \\ ${ }^{c}$ Leipzig University, Neumarkt 9-19, D-04109 Leipzig, Germany \\ ${ }^{\mathrm{d}}$ University of Münster, Fliednerstraße 21, 48149 Münster, Germany \\ ${ }^{\mathrm{e}}$ University of Applied Sciences Magdeburg-Stendal, Osterburgerstraße 25, 39576 Stendal, Germany
}

\section{A R T I C L E I N F O}

\section{Keywords:}

EEG

Omission

Predictive coding

Auditory N1

Children

\begin{abstract}
A B S T R A C T
Action is an important way for children to learn about the world. Recent theories suggest that action is inherently accompanied by the sensory prediction of its effects. Such predictions can be revealed by rarely omitting the expected sensory consequence of the action, resulting in an omission response that is observable in the EEG. Although prediction errors play an important role in models of learning and development, little is known about omission-related brain responses in children.

This study used a motor-auditory omission paradigm, testing a group of 6-8-year-old children and an adult group $(\mathrm{N}=31$ each). In an identity-specific condition, the sound coupled to the motor action was predictable, while in an identity unspecific condition the sound was unpredictable.

Results of a temporal principal component analysis revealed that sound-related brain responses underlying the N1-complex differed considerably between age groups. Despite these developmental differences, omission responses (oN1) were similar between age groups. Two subcomponents of the oN1 were differently affected by specific and unspecific predictions.

Results demonstrate that children, independent from the maturation of sound processing mechanisms, can implement specific and unspecific predictions as flexibly as adults. This supports theories that regard action and prediction error as important drivers of cognitive development.
\end{abstract}

\section{Introduction}

Infants and children exhibit a strong desire to act in the world. In their first year of life, children voluntarily and repeatedly drop objects, for instance a spoon, and listen highly concentrated to the sound of the impact. A growing body of empirical findings demonstrates the importance of such behaviour, showing that action execution forms associations between motor acts and sensory consequences, which are subsequently used to improve perception (Adolph and Hoch, 2019; Hunnius and Bekkering, 2014). Despite the important role of action-effect couplings in development, the maturation of the psychophysiological mechanisms behind this process, their flexibility and specificity, and their interaction with sensory processing are largely unknown. More specifically, the effect of the large developmental changes in the auditory system in middle childhood and its relation to neuronal mechanisms underlying action-effect couplings is largely unexplored.
From a psychophysiological perspective, action-effect couplings are often considered in the context of predictive coding. This theory hypothesises that higher-level cortical areas send sensory predictions downwards to lower levels. When the predicted input does not match the actual sensory input, a prediction error is generated which is propagated back up the hierarchy, acting as information to update models and generate better predictions (Friston, 2005). An action that is reliably coupled to a sensory consequence (such as dropping a spoon and hearing its impact on the floor) propagates sensory predictions downwards to sensory levels (Arnal and Giraud, 2012). At the moment the action is performed, sensory predictions are compared to actual input, where incorrect predictions result in prediction errors that are propagated back up to higher levels. For infants and children, these prediction errors might be a crucial source of information for learning and development (Emberson, 2017; Köster et al., 2020; Trainor, 2012).

An intriguing example of action-induced prediction error is the electrophysiologically recorded omission response elicited by motor-

\footnotetext{
* Corresponding author at: Leibniz Institute for Neurobiology, Brenneckestraße 6, 39118 Magdeburg, Germany.

E-mail address: Tjerk.Dercksen@lin-magdeburg.de (T.T. Dercksen).
} 
auditory couplings (Dercksen et al., 2020, from the same lab as the current study; SanMiguel et al., 2013a, 2013b). In these experiments, subjects had to press a button every $600-1200 \mathrm{~ms}$, which immediately resulted in a sound most of the time, but where occasionally the sound was unexpectedly omitted. Predictive coding hypothesises that in these cases, where an auditory prediction is present but auditory input is absent, a prediction error should be elicited in auditory areas. Indeed, a cascade of omission responses is visible in the EEG, where early responses likely originate from auditory areas (SanMiguel et al., 2013b). Similar omission responses have been observed in visual-auditory paradigms (Stekelenburg and Vroomen, 2015; Van Laarhoven et al., 2017). Omission paradigms are a strong tool to investigate prediction as they avoid confounding adaptation effects and can be regarded to solely reflect prediction (error) related activity (SanMiguel et al., 2013b; Schröger et al., 2015). The lack of bottom-up sensory input in these paradigms is particularly beneficial when studying cognitive development, where stimulus processing is still immature (e.g., for auditory processing: Wunderlich and Cone-Wesson, 2006) and could therefore confound inferences regarding the maturation of prediction processes. Several other developmental studies have already demonstrated the benefits of an omission approach using fNIRS (Boldin et al., 2018; Emberson et al., 2015), pupil dilation (Zhang et al., 2019), and EEG (Winkler et al., 2009). These studies were carried out with infants and did not apply motor-sensory couplings that require action. Instead, passive couplings were used, for instance combinations of audio-visual stimuli where occasionally visual stimuli were omitted (e.g. Boldin et al., 2018).

In the paradigm of SanMiguel et al. (2013a, 2013b) and Dercksen et al. (2020) the earliest omission-related component in the event-related potential (ERP) is the omission N1 (oN1). As the first indication of (cortical) prediction error, and given its presumed origin in the auditory cortex, the oN1 likely reflects the unconscious comparison between sensory prediction and sensory input (Arnal and Giraud, 2012; Friston, 2005; Knolle et al., 2012; Schröger et al., 2015). According to predictive coding models of perception, the oN1 is related to the N1-family of sound evoked ERP components, as prediction errors are thought to be generated by the sensory processing hierarchy responsible for sound perception (Bastos et al., 2012; Bendixen et al., 2012; Schröger et al., 2015). Significant developmental changes in the sound evoked N1 subcomponents can be observed in middle childhood. The vertex N1 (also termed N1b) takes a long time to fully develop, showing considerable changes until adolescence (Čeponien et al., 1998, 2002; Eggermont and Ponton, 2003; Ponton et al., 2000;). N1b is often absent in middle childhood, where normally the P1 is the dominant component of the ERP (e.g., Silva et al., 2017; Wetzel and Schröger, 2007), and is sensitive to stimulus presentation and the physical properties of the stimulus (Näätänen and Picton, 1987). The temporal N1 subcomponents-which are considered part of the T-complex (Na and $\mathrm{Tb}$ )are also subject to significant changes throughout development (Rinker et al., 2017; Shafer et al., 2015). The first negative peak, the Na (also termed N1a), is observed in 18-month-olds and is assumed to decrease in both amplitude and latency as the brain matures throughout childhood (Tonnquist-Uhlen et al., 2003; Wunderlich and Cone-Wesson, 2006). The N1a is assumed to reflect the activation of neural generators underlying stimulus detection (Näätänen and Picton, 1987). The later Tb (also termed N1c) emerges after three years of age and decreases in amplitude with age (Albrecht et al., 2000; Ponton et al., 2002), but effects on latency are inconsistent (Albrecht et al., 2000; Mahajan and McArthur, 2013; Ponton et al., 2002). The N1c is assumed to reflect the activation of neural generators underlying stimulus discrimination (Näätänen and Picton, 1987). Generally, the N1 complex is thought to reflect sensory processing (Joos et al., 2014) and is mostly generated by the primary and secondary auditory cortices and auditory association areas (Picton et al., 1999; Woods, 1995).

Given the significant developmental changes of N1 subcomponents, and the discussed relation between auditory omission responses and sound evoked processes, it is unclear whether the oN1 undergoes similar developmental changes. In a broader sense, it is uncertain whether motor-sensory omission responses are present at all in children given the scarce research regarding the development of the responsible prediction pathways. Basirat et al. (2014) found that 3-month-old infants already demonstrate distinct error responses to local sound (deviation from directly successive stimuli, e.g. AAAB, eliciting an early response) and global sound (general rules over multiple sequences, e.g. AAAB AAAB AAAA, eliciting a late response) violations, suggesting that the hierarchical elicitation of prediction error is present from a young age. The responses were, however, very different from adult responses in terms of latency, amplitude, polarity and topography, which might suggest an ongoing development of these components similar to sound evoked processes. Furthermore, action-effect associations have been demonstrated behaviourally in infants around the end of the first year of life (Elsner, 2007). Paulus et al. (2012) additionally observed motor-related EEG activity in 8-month-olds in response to a sound that was associated with the movement of a toy. These findings suggest that action-effect couplings are present from an early age. However, it is unclear whether violations of these couplings elicit sensory error responses in school age children that resemble those of adults given the involvement of an immature auditory system. In particular, the dissociation between the generation of sensory predictions that are specific for the identity of an auditory event, and more general predictions are barely investigated, even in older children. This knowledge gap regarding the development of action-effect couplings is especially notable given the essential role they play in models of motor control (Shadmehr et al., 2010; Imamizu, 2010), speech production (Hickok and Poeppel, 2007; Pickering and Garrod, 2013), and ideomotor theories in general (for a review see Shin et al., 2010).

The current study aims to investigate the early prediction error (oN1) elicited by motor-auditory couplings, using a child-friendly version of the omission paradigm of SanMiguel et al. (2013a) and Dercksen et al. (2020) that until now was performed in adults only. The study measures a child group aged 6-8 years and an adult control group. This age range ensured that children on the one hand were able to perform the task, and on the other hand demonstrate a comparable, immature brain response to sounds. Our paradigm tests motor-auditory omission responses in two conditions: one where the identity of the sound is known (single sound condition) and one where the identity of the sound is unknown (random sound condition). Dercksen et al. (2020) showed that an oN1 is elicited in both conditions, but that the response is stronger when the sound can be completely predicted (single sound condition). They attributed these findings to possible precision weighting effects: a highly specific prediction (single sound condition) increases the weight that is attributed to the error units, while an unspecific prediction (random sound condition) decreases the weight (Feldman and Friston, 2010). It is unknown whether the children's auditory cortical system, that is not matured in the respective age range, can already differentiate between identity specific and unspecific predictions in a similar way as adults. Therefore, the current study can determine 1) if the oN1 is elicited in children especially given the developmental processes of its sound evoked counterparts - and 2) whether similar precision weighting effects can also be observed in children.

\section{Material and methods}

\subsection{Participants}

EEG and behavioural data were acquired for a group of adults and a group of children. A total of 39 children and 32 adults were measured. 9 participants were excluded ( 8 children, 1 adult). 2 children were excluded because they could not perform the task well enough (defined as pressing more than 150 times-out of 1760 trials-too early/late), 3 because of technical problems, and 3 because children switched buttonpress hand repeatedly during the experiment. 1 adult was excluded 
because of repeatedly falling asleep and not pressing the button anymore. 31 children were regarded for further analysis ( 21 female; age range: $6-8$; mean age $=7.9, \mathrm{SD}=0.7$ years; 2 left-handed as measured by an adapted German version of the Oldfield Scale; Oldfield, 1971; left-handed participants performed experiment with right hand). 31 adults were regarded for further analysis (12 female; age range: $20-35$; mean age $=25.3, \mathrm{SD}=4.5$ years; 2 left-handed as measured by an adapted German version of the Oldfield Scale; Oldfield, 1971; left-handed participants performed experiment with right hand). All participants reported normal hearing and were compensated with money (for adults) or a voucher for a children's shop (for children). Adults gave written consent prior to the experiment, whereas in the case of children both children and parents gave written consent. For children, it was vocally explained that (translated from German): "I participate voluntarily and if I am tired or would like to stop doing the experiment, it is perfectly ok and we can stop." If the child agreed, they would write their name at the bottom of the form that had the vocally mentioned statement written on it. The project was approved by the local ethical committee.

\subsection{Stimuli}

48 different common environmental sounds (e.g., dog, car-horn, trumpet) rated as identifiable by an independent sample of participants (in $200 \mathrm{~ms}$ form, see Wetzel et al., 2011) were used as sound stimuli. Sounds were presented binaurally for $200 \mathrm{~ms}$ and were tapered-cosine windowed (10 ms rise- and $10 \mathrm{~ms}$ fall-time) and root mean square (RMS) matched. Loudness was set at 70.4 dB SPL for all participants. While performing the experiment, participants were asked to watch a children's movie that was played silently on a screen at $\sim 60$ $\mathrm{cm}$ distance from the participants eyes $\left(10.3^{\circ} \times 18.9^{\circ}\right.$ visual angle $)$. The movie (Burton and Starzak, 2015) was a clay-animated story about the adventures of a sheep, that was easy to understand without sound (as there was no speech involved) and suitable for the young age group.

\subsection{Apparatus}

Participants were seated in a dimly lit, electrically shielded, and acoustically attenuated chamber, while EEG was continuously recorded. The experiment was programmed using Psychtoolbox (version 3.0.15; Brainard, 1997) and ran on a Linux-based system using GNU Octave (version 4.0.0). Auditory stimuli were presented using Sennheiser HD-25 headphones. Visual stimuli were presented using a VIEWPixx/EEG Display (Resolution 1920(H) × 1080(V) - 23.6-in. display size). To ensure the button did not make a sound when pressed, a custom-built infra-red photoelectric button was used that was additionally padded with sound absorbing material.

\subsection{Task and procedure}

Participants sat approximately $60 \mathrm{~cm}$ from a screen, having their right index finger on a button. In all conditions, participants were asked to press a button every $600-1200 \mathrm{~ms}$, while at the same time watch a movie. If the button was pressed too quickly $(<600 \mathrm{~ms}$ ISI) the movie was interrupted and a bunny would appear. If the button was pressed too slowly ( $>2000 \mathrm{~ms}$ ISI) the movie was interrupted and a snail would appear. As soon as the bunny/snail disappeared, participants could resume the experiment with the next button press. Two distinct sound conditions (single sound, random sound) and a motor control condition were presented. In the sound conditions, a button press resulted in a sound (without delay) $85 \%$ of the time, where the remaining $15 \%$ of trials were unexpectedly omitted. Sound blocks had 220 sound trials and 40 omission trials, and motor control blocks had 100 trials. Omissions were randomly placed, under the restricting conditions that the first five trials of every block were always sound trials, and every two trials following an omission were always sound trials. In the single sound condition, the same sound was presented in all sound trials of the block. Different sounds were used as the single sound in separate blocks, where all 48 sounds were balanced across participants. In the random sound condition, sounds changed on every trial. A no-sound motor control condition was included in which no sound was presented with the button press to be able to subtract the neural activity related to the pressing of the button. Before the experiment, a few short training blocks were completed where subjects attempted to press the button every 600-1200 ms, using an intuitive feedback display. In the first training block, a speedometer was presented on the screen, where a pointer would move after every button press either in a green (correct time between button presses, middle area of the speedometer) or red (too slow/fast between button presses, left/right areas of the speedometer) area of the speedometer. This training block presented 150 trials without sound. Subsequently, three short training blocks of 20 trials were presented to familiarise participants with the motor control, single, and random sound conditions together with the movie. Training blocks were repeated when necessary (i.e., when the participant did not consistently press in the correct rhythm yet, or when the participant wanted more practice). What followed were 8 experimental blocks ( 3 single sound, 3 random sound, 2 motor control). The order of the blocks was completely randomised. Blocks were approximately 3 min long. A total of 660 sound trials and 120 omission trials were performed for each sound condition, and 200 trials were performed as no-sound motor control. Total experiment time was about $45 \mathrm{~min}$ including breaks.

\subsection{Data recording}

EEG was recorded from a total of 31 active electrodes, placed according to the extended international 10-20 system at the following positions: FP1, Fz, F3, F7, FC5, FC1, C3, T7, CP5, CP1, Pz, P3, P7, Oz, P4, P8, CP6, CP2, Cz, C4, T8, FC6, FC2, F4, F8, FP2, and the left (M1) and right (M2) mastoids. Furthermore, EOG was recorded from three electrodes placed left and right of the outer canthi of the eyes and below the left eye. The reference electrode was placed on the tip of the nose. An Actichamp amplifier (BrainProducts, Gilching, Germany) was used, recording at $500 \mathrm{~Hz}$ using Vision Recorder software (version 1.21).

\subsection{EEG data preprocessing}

EEG data analysis was performed with MATLAB software using the EEGLAB toolbox (Delorme and Makeig, 2004). Data was filtered offline with a $0.1 \mathrm{~Hz}$ high-pass filter (Kaiser windowed sinc FIR filter, order = 8024 , beta $=5$, transition band width $=0.2 \mathrm{~Hz}$ ) and a $48 \mathrm{~Hz}$ low-pass filter (Kaiser windowed sinc FIR filter, order $=402$, beta $=5$, transition band width $=4 \mathrm{~Hz}$ ). Data was segmented into epochs starting 200 $\mathrm{ms}$ before and ending $500 \mathrm{~ms}$ after button press. All trials outside the 600-2000 ms button-press time limit were excluded. Noisy channels were removed from the data, which were defined as having a robust z-score of the robust standard deviation larger than 3 (Bigdely-Shamlo et al., 2015). These channels were removed from analysis and interpolated after ICA. Epochs exceeding a $500 \mu \mathrm{V}$ signal-change per epoch threshold were removed. ICA was performed to correct for artefacts. This was done on raw data that was $1 \mathrm{~Hz}$ high-pass filtered (Kaiser, order $=1604$, beta $=5$, transition band width $=1 \mathrm{~Hz}$ ) and $48 \mathrm{~Hz}$ low-pass filtered (same as above), as $1-2 \mathrm{~Hz}$ high-pass filters improve ICA performance (Klug and Gramann, 2020; Winkler et al., 2015). After ICA, data was segmented -200 to $500 \mathrm{~ms}$ around the button press. The same channels and trials were removed as was done in the previous step. The obtained demixing matrix was subsequently applied to the $0.1-48$ $\mathrm{Hz}$ filtered data. Artefact ICs were detected with support of the IClabel plugin (Pion-Tonachini et al., 2019). Two independent raters judged components, aiming to remove all heart-, eye- and muscle-related components. Selected components were then discussed to come to a final judgement of components to be removed. For children, on average 11 components were rejected (median $=11, \min / \max =8 / 16, S D=2$ ), 
while for adults on average 10 components were rejected (median $=9$, $\min / \max =6 / 15, S D=2$ ). Each epoch was baseline corrected by subtracting the mean amplitude of the -150 to -50 ms window preceding stimulus onset. Finally, the first five trials of each block, the two trials following an omission, and trials that exceeded $125 \mu \mathrm{V}$ signal-change per epoch were excluded from analysis. Individual ERPs were computed for each condition and every subject.

\subsection{Behavioural data}

Behavioural data were analysed to determine if there were systematic differences between age groups in the number of trials pressed outside the appropriate time window and in the rhythm that the button was pressed. This rhythm was determined based on the behavioural data from which any too early/late button presses were removed. Trials were defined as too early when time between button presses was less than $600 \mathrm{~ms}$, and as too late when time between button presses exceeded more than $2000 \mathrm{~ms}$ (the same time window that would result in an interruption of the experiment in case of violation, see Section 2.4).

\section{8. $P C A$}

Temporal PCA was used to analyse ERPs. This method aims to statistically decompose ERP waveforms into their constituent building blocks (see Dien, 2012 for a tutorial). PCA is particularly suited for the investigation of ERPs in developmental populations reducing problems due to the enhanced noise level (Dien, 2012). Given the typically different component structure and latencies in the child and adult groups, PCAs were performed separately for the adult and child group and for the sound and omission conditions (see Barry et al., 2016 for a demonstration that this approach is superior with systematic component latency differences). The number of retained components was determined using Horn's parallel test. An R implementation of the Component loss rotation (Jennrich, 2004a, 2004b, 2006) method with Kaiser normalisation was applied to the initial PCA solution as described by Scharf and Nestler (2019). Component loss rotation is substantially less prone to conflating components with strong temporal and spatial overlap than other rotation methods. This property made component loss rotation especially appropriate since we were specifically interested in a good decomposition of components in the N1 time range. Two separate PCAs were computed on both age groups, one on the individual average ERP responses to sound omissions (plus motor-control) and one on the individual average ERP responses to sounds (plus motor-control). The PCA of omission responses (plus motor-control) focused on the analysis of the oN1, which was recognised on the basis of its typical temporal and topographical characteristics (as described, e.g., by Dercksen et al., 2020). This PCA was computed on the individual averages of the motor control, single sound omissions, and random sound omissions. The ROI for statistical testing of the oN1 was based on the topography reported in previous studies (SanMiguel et al., 2013a, 2013b; Dercksen et al., 2020), using electrodes T7 and T8 (which were the only electrodes that were present in all three aforementioned studies). The PCA of sound responses (plus motor-control) focused on analysis of the sound ERP. This PCA was computed on the individual averages of the motor control, single sounds, and random sounds. ROIs for the components were based on the topography of activations. For children, frontal P1: F3, Fz, F4, FC1, FC2 (frontal/central); T7, T8 (temporal). Centro-lateral P1: FC5, FC1, FC2, FC6; T7, T8. N1c/P2: FC1, FC2, Cz; T7, T8. For adults, P1: F3, Fz, F4, FC1, FC2; T7, T8. N1b: C3, Cz, C4; T7, T8. N1c: F3, Fz, F4, FC1, FC2; T7, T8. P2: FC1, FC2, Cz; T7, T8. The results of the PCAs of sound-related ERPs were only analysed within groups.

Because separate PCAs for omission responses were performed for the adult and child group, analysing the differences between the two groups could not be performed on the level of the factor scores and required further consideration. First, we determined the oN1 components as described above separately for each group. Then, we reconstructed the omission PCA component time courses in $\mu \mathrm{V}$-units reflecting the appropriately scaled contribution of each component to the observed ERP (as previously applied for example by Bonmassar et al., 2020) per component, participant, electrode location, and condition, by multiplying the component score by the peak amplitude of the PCA component loading times the standard deviation (per time point). This conversion of component loadings to real world units $(\mu \mathrm{V})$ was demonstrated by Dien (1998, Appendix for a formal proof; 2012 for an accessible explanation). The resulting time course reflects the portion of the recorded waveform accounted for by each component scaled to $\mu \mathrm{V}$, allowing a statistical comparison between the adult and child groups. An amplitude measure for the oN1 components was obtained by the peak amplitude for the single and random condition, respectively, in each group. Before the amplitudes were subjected to comparisons between age groups, the motor-control waveform was subtracted from the motor-auditory waveform to correct for the contribution of motor activity to the ERP.

\subsection{Statistical analyses}

Behavioural data was tested for differences between groups regarding the number of trials pressed outside the appropriate time window and the time asynchrony between button presses. Both measures were tested using frequentist and Bayesian independent $t$-tests.

The early and late subcomponents of the oN1 were tested for differences between conditions using separate paired samples $t$-tests (single omission vs. motor control, random omission vs. motor control, single omission vs. random omission) within each age group. As previous literature provides clear hypotheses about the expected effects, $t$-tests were performed one-sided. Equivalent comparisons were tested using Bayesian paired samples t-tests (single omission vs. motor control, random omission vs. motor control, single omission vs. random).

Differences between groups were tested using a mixed model ANOVA on the omission minus motor control difference amplitudes testing for elicitation of the components (intercept; within subject), for condition differences within groups (condition: single vs. random condition; within subject), and for amplitude differences between groups (age group: children vs. adults; between subject). A significant interaction effect of condition $\times$ age group would indicate between group differences for the condition effects. All mixed model ANOVA main and interaction effects including the intercept term (reflecting component elicitation) were tested with the corresponding Bayesian $t$-tests. This analysis strategy provided optimal correspondence between frequentist and Bayesian tests for evaluating the support provided by the data for the alternative and null hypotheses. Effect size was reported using the generalised $\eta^{2}\left(\eta_{g}^{2}\right.$; Bakeman, 2005).

Bayesian paired samples $t$-tests corresponding to the mixed model ANOVA main effects and interactions were performed in $\mathrm{R}$ using the BayesFactor package (Morey and Rouder, 2018). The null hypothesis corresponded to a standardised effect size $\delta=0$, while the alternative hypothesis was defined as a Cauchy prior distribution centred around 0 with a scaling factor of $\mathrm{r}=0.707$ (the default "medium" effect size prior scaling). Resulting Bayes Factors $\left(B F_{10}\right)$ were interpreted following Lee and Wagenmakers (2013), who give the labels anecdotal (0.33-3), moderate (3-10 or $0.33-0.1)$, strong (10-30 or $0.1-0.033)$, and very strong ( $>30$ or $<0.033$ ) for specific ranges of the Bayes Factor. We replaced the label "anecdotal" with "weak" and "very strong" with "decisive" to aid interpretation. Analyses were conducted using R 3.6.1 (R Core Team, 2014).

Sound evoked components were tested for differences between conditions using separate paired samples $t$-tests (single omission vs. motor control, random omission vs. motor control, single omission vs. random omission) within each age group. These $t$-tests were performed two-sided. Equivalent comparisons were tested using Bayesian paired samples $t$-tests (single omission vs. motor control, random omission vs. motor control, single omission vs. random). 


\section{Results}

In general, both adults and children were able to press the button within the appropriate time window, although children made more mistakes than adults. The average number of trials pressed outside the time window in the child group was 42 (median $=34, \min / \max =3$ / $145, \mathrm{SD}=38$ ) and in the adult group 3 (median $=1, \min / \mathrm{max}=1 / 15$, $\mathrm{SD}=3)$. The observed data provides decisive evidence for a difference between groups $\left(B F_{10}=3.88 \times 10^{4}, d=-1.46\right.$ [95\% CI: $\left.-2.02-0.90\right]$, $t(60)=-5.757, p<.001)$. Mean inter-press interval in the child group was $941 \pm 123$ (SD) and in the adult group $960 \pm 130$ (SD). The observed data provides moderate evidence against a difference between groups $\left(B F_{10}=0.30, d=-0.15\right.$ [95\% CI: $\left.-0.650 .35\right], t(60)=-0.584$, $p=0.561)$.

The PCA of omission responses extracted a total of 13 components in the children group, and 15 components in the adult group. The PCA of sound responses extracted a total of 10 components in the children group and 13 components in the adult group (Fig. 1).

\subsection{Early oN1}

PCA extracted two components in the oN1 time range, an early and a late component, in both age groups (see Table 1 for mean amplitudes). In the child group, PCA component 2 presumably reflected the early oN1 component, explaining $11.7 \%$ of variance. It peaked at $130 \mathrm{~ms}$ and was maximal over temporal leads (see Fig. 1 for overview of component loadings, Fig. 2 for component details). In the adult group, PCA component 5 presumably reflected the early oN1 component, explaining
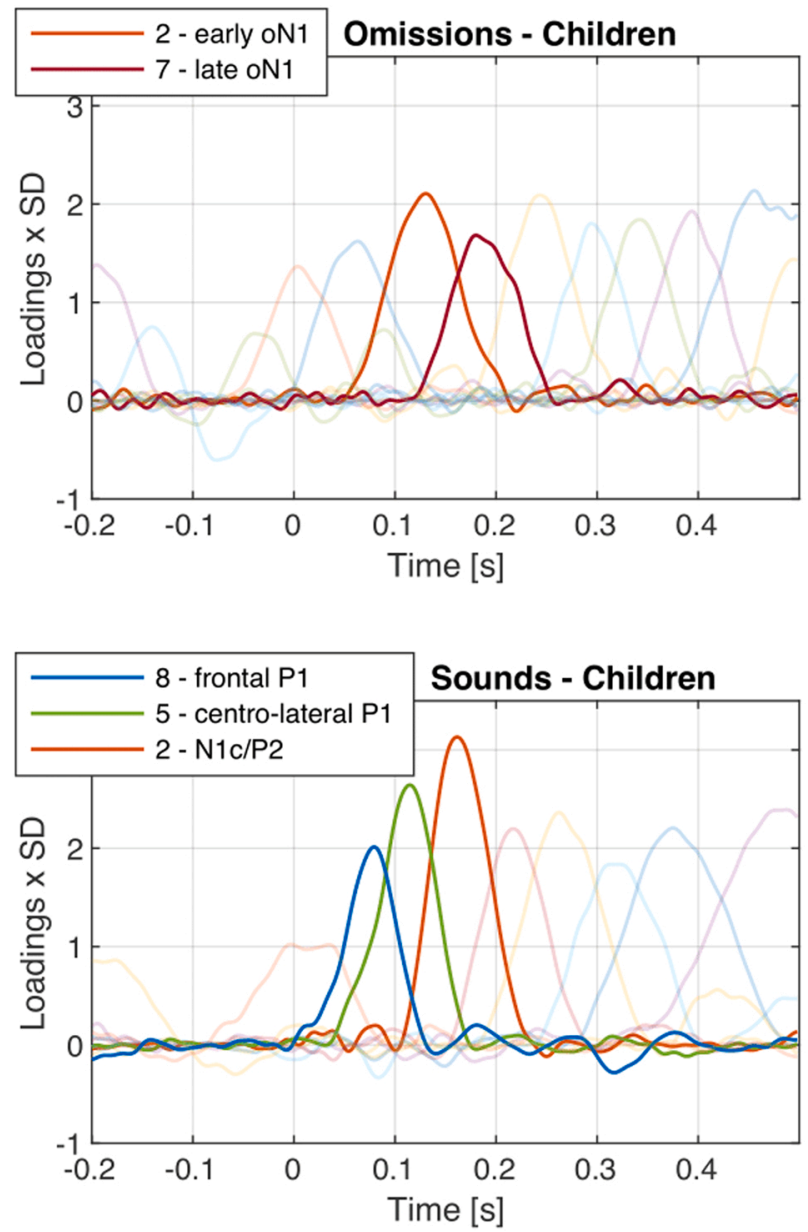

Table 1

Motor corrected early and late oN1 amplitudes in the single (SO-M) and random sound conditions (RO-M) in $\mu \mathrm{V}$ units incl. [95\% CI] in children and adults.

\begin{tabular}{cllll}
\hline & & Adults & Children & Mean \\
\hline Early & SO-M & $-0.55[-0.77$ & $-1.60[-2.21$ & $-1.08[-1.42$ \\
oN1 & & $-0.33]$ & $-1.00]$ & $-0.73]$ \\
& RO- & $-0.27[-0.44$ & $-0.92[-1.48$ & $-0.59[-0.89$ \\
& M & $-0.09]$ & $-0.36]$ & $-0.29]$ \\
& Mean & $-0.41[-0.55$ & $-1.26[-1.77$ & \\
& & $-0.27]$ & $-0.75]$ & \\
Late & SO-M & $-0.67[-0.93$ & $-1.22[-1.78$ & $-0.94[-1.25$ \\
oN1 & & $-0.40]$ & $-0.66]$ & $-0.63]$ \\
& RO- & $-0.50[-0.80$ & $-0.74[-1.34$ & $-0.62[-0.94$ \\
& M & $-0.19]$ & $-0.13]$ & $-0.29]$ \\
& Mean & $-0.58[-0.81$ & $-0.98[-1.47$ & \\
& & $-0.35]$ & $-0.49]$ & \\
\hline
\end{tabular}

$8.9 \%$ of variance. In adults, the early oN1 component peaked at $90 \mathrm{~ms}$ and was maximal over temporal leads (see Fig. 1 for overview of component loadings, Fig. 2 for component details).

The early oN1 component was elicited with comparable topography (maximal at temporal leads) across age groups (see Fig. 2). Early oN1 amplitude was larger in children compared to adults and larger in the single compared to the random sound condition. The observed data provide strong evidence for the elicitation of the early oN1 component (intercept term: $B F_{10}=6.56 \times 10^{5}, d=0.82$ [95\% CI: 0.531 .11$], F$ $\left.(1,60)=41.78, p<.001, \eta_{\mathrm{g}}{ }^{2}=0.336\right)$. Furthermore, the data provide strong evidence for higher early oN1 amplitudes in children compared to adults (main effect age group: $B F_{10}=20.54, d=0.84$ [95\% CI: 0.29
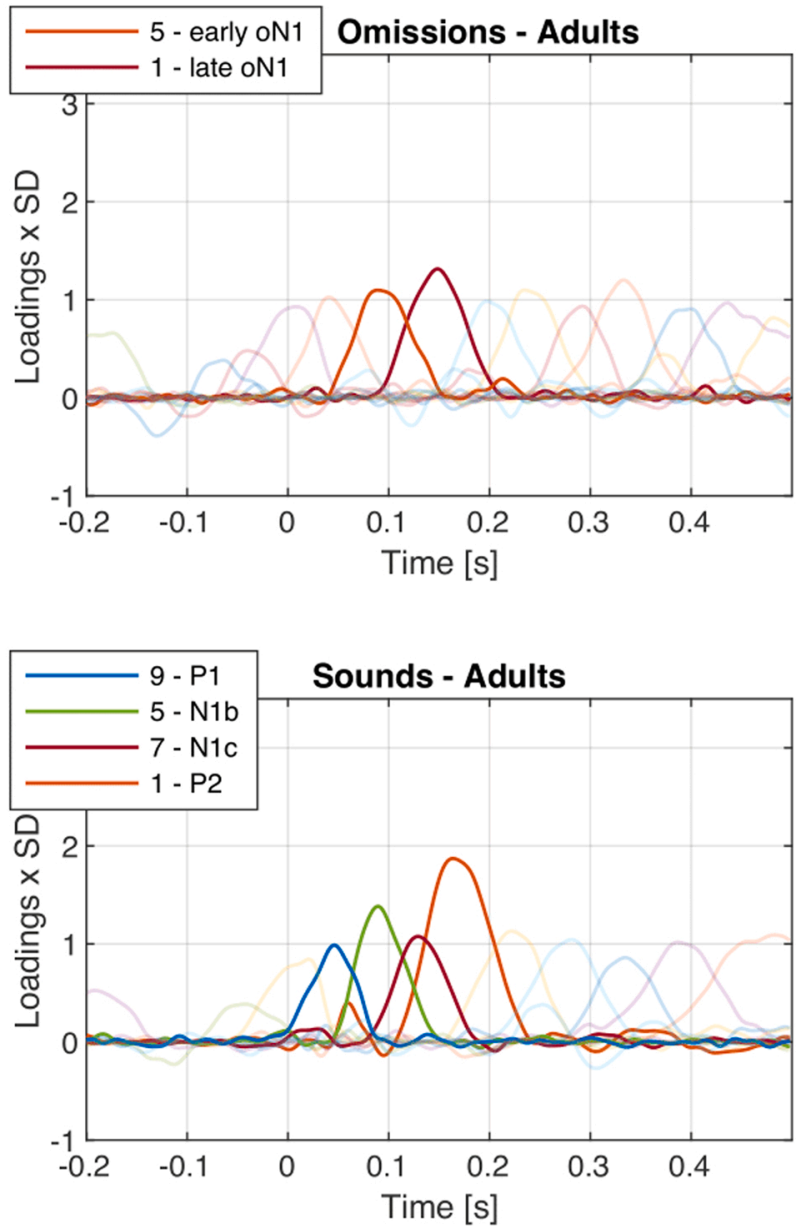

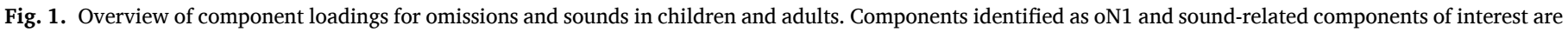
shown in opaque. 
A Omission ERPs - Children

Early oN1

A1 (130 ms; comp. 2)

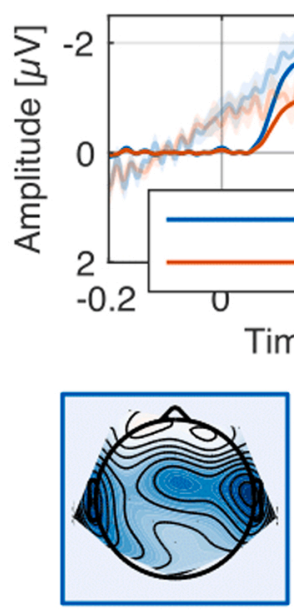

Time [s]
2)

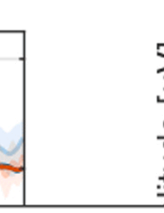

Single sound omisson minus motor Random sound omission minus motor

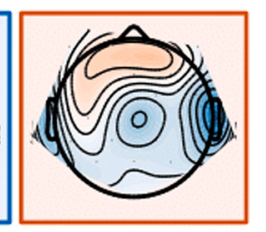

Late oN1

A2 (180 ms; comp. 7)

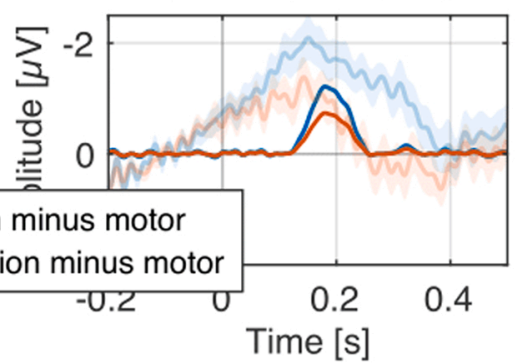

Fig. 2. Early and late oN1 components in children and adults. Panel A: Reconstructed PCA component difference waveforms (opaque) and grand-average difference waves plus $95 \%$ CIs (transparent) for single (blue) and random (red) sound conditions for electrodes T7 \& T8. Panel B: Omission minus motor control difference component topographies at component peak latencies (as reported in panel A) in the single (left column) and random sound conditions (right column). The omission response can be seen over temporal areas.

\section{B Omission ERPs - Adults}
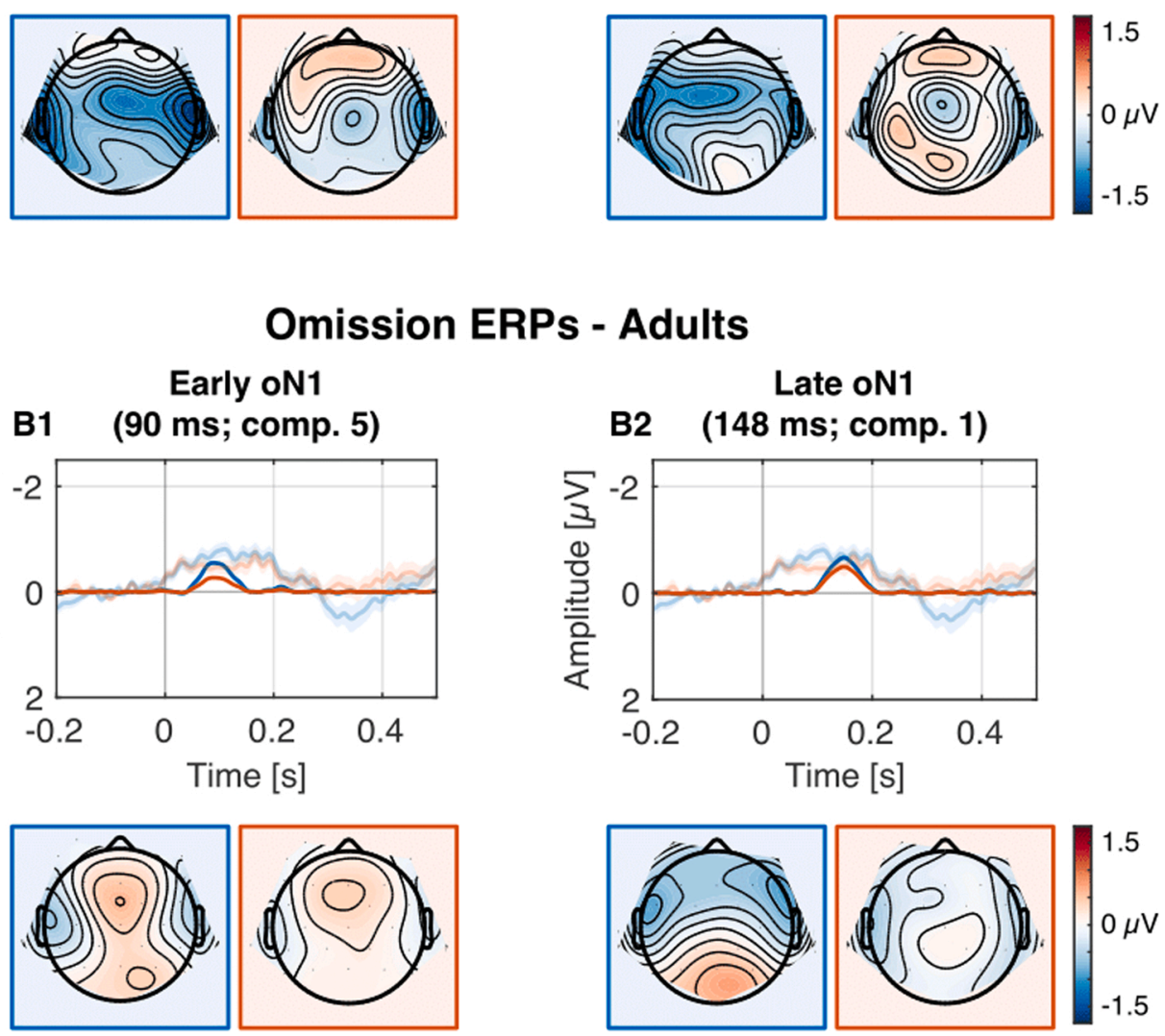

1.5

$0 \mu \mathrm{V}$

$-1.5$

1.37], $\left.F(1,60)=10.89, p=0.002, \eta_{\mathrm{g}}{ }^{2}=0.117\right)$ and moderate to strong evidence for higher early oN1 amplitudes in the single sound compared to the random sound condition (main effect condition: $B F_{10}=9.17$, $d=0.39$ [95\% CI: 0.130 .65 ], $F(1,60)=9.37, p=0.003, \eta_{\mathrm{g}}{ }^{2}=0.041$ ). The data indicated that the amplitude difference between single and random sound conditions is similar rather than different between the age groups, however, the evidence was not conclusive. If the condition effect is modulated by age group the interaction effect is expected to be small (interaction effect of condition $\times$ age group: $B F_{10}=0.52, d=0.33$ [95\% CI: -0.180 .83 ], $\left.F(1,60)=1.66, p=0.203, \eta_{\mathrm{g}}{ }^{2}=0.007\right)$.

\subsection{Late oN1}

In the child group, PCA component 7 presumably reflected the late oN1 component, explaining $8.7 \%$ of variance (see Table 1 for mean amplitudes). In children, the late oN1 component peaked at $180 \mathrm{~ms}$ and was maximal over temporal leads (see Fig. 1 for overview of component loadings, Fig. 2 for component details). In the adult group, PCA component 1 presumably reflected the late oN1 component, explaining
$11.4 \%$ of variance. In adults, the late oN1 component peaked at $148 \mathrm{~ms}$ and was maximal over temporal leads (see Fig. 1 for overview of component loadings, Fig. 2 for component details).

A late oN1 component was elicited with comparable topography (maximal at temporal leads) across age groups (see Fig. 2). Late oN1 had similar amplitudes in children compared to adults and in the single compared to the random sound condition. The observed data provide strong evidence for the elicitation of the late oN1 component (intercept term: $B F_{10}=8.56 \times 10^{4}, d=0.75$ [95\% CI: 0.471 .03$], F(1,60)=$ $\left.34.94, p<.001, \eta_{\mathrm{g}}{ }^{2}=0.287\right)$. The data suggest that late oN1 amplitudes were similar rather than different between children and adults (main effect age group: $B F_{10}=0.67, d=0.38$ [95\% CI: -0.130 .89 ], $F(1,60)=$ 2.27, $p=0.137, \eta_{\mathrm{g}}{ }^{2}=0.026$ ) and also between single sound and random sound conditions (main effect condition: $B F_{10}=0.69, d=0.24$ [95\% CI: -0.020 .49 ], $\left.F(1,60)=3.43, p=0.069, \eta_{g}{ }^{2}=0.017\right)$ but the evidence was not conclusive. Potential age and condition effects are expected to be small if any. The data provide weak evidence that the amplitude difference between single and random sound conditions is similar rather than different between the age groups (interaction effect 
of condition $\times$ age group: $B F_{10}=0.359, d=0.22$ [95\% CI: -0.280 .72 ], $\left.F(1,60)=0.77, p=0.383, \eta_{\mathrm{g}}^{2}=0.004\right)$.

\subsection{Sound-related ERPs}

The morphology and amplitude of the ERPs evoked by sounds differed substantially between age groups. In adults - after motor correction - the standard P1-N1-P2-N2 morphology was observed. Here, PCA extracted two N1 components (Fig. 3 panel B2/B3) presumably reflecting a vertex $\mathrm{N} 1 \mathrm{~b}(90 \mathrm{~ms})$ and a temporal $\mathrm{N} 1 \mathrm{c}(128 \mathrm{~ms})$. In children, in the same time range, a large positive wave is observed, which PCA subdivides in three separate components (Fig. 3 panel A): a frontal positive wave at $80 \mathrm{~ms}$ (termed frontal P1), a centro-lateral positive wave at $114 \mathrm{~ms}$ (termed centro-lateral P1), and a central positive wave at $162 \mathrm{~ms}$ (termed P2). At temporal leads, these components resemble parts of the T-complex (Wolpaw and Penry, 1975): the frontal $\mathrm{P} 1$ component inverts polarity at temporal leads and resembles the $\mathrm{Na}$ or $\mathrm{N} 1 \mathrm{a}$, the centro-lateral P1 resembles the positive Ta component, and the P2 inverts polarity resembling the Tb or N1c. None of children's components reflected characteristics of the vertex N1b observed in adults.
A

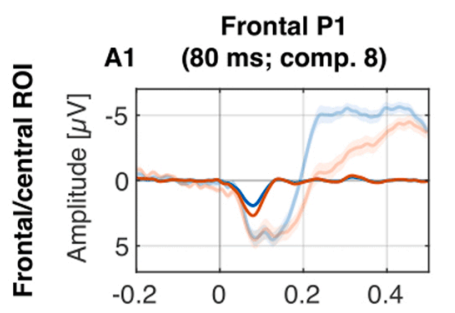

뭄

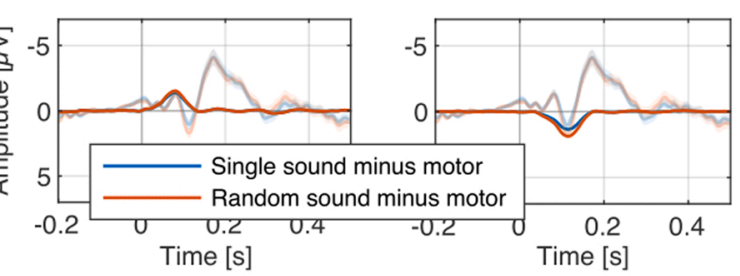

\section{Sound ERPs - Children}

\section{A2 Centro-lateral P1}

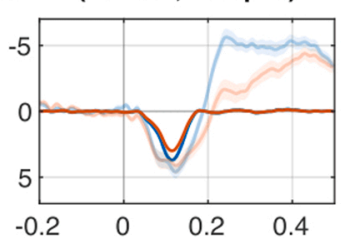

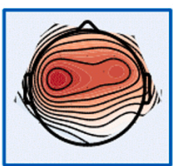

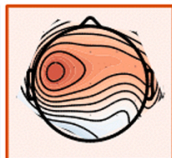

N1c/P2

A4 (162 ms; comp. 2)
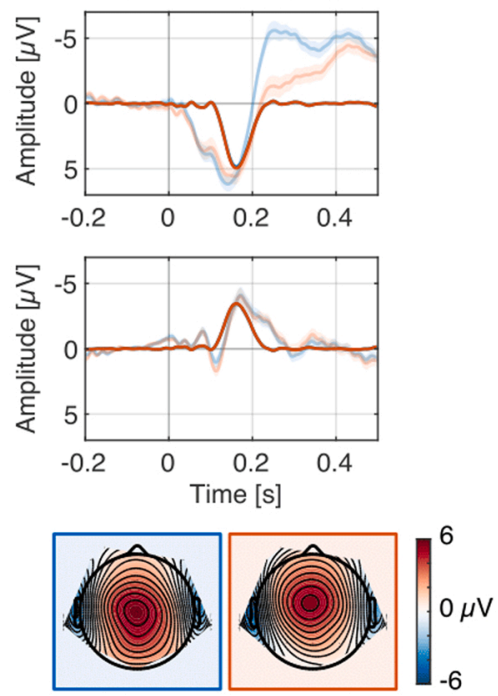

B
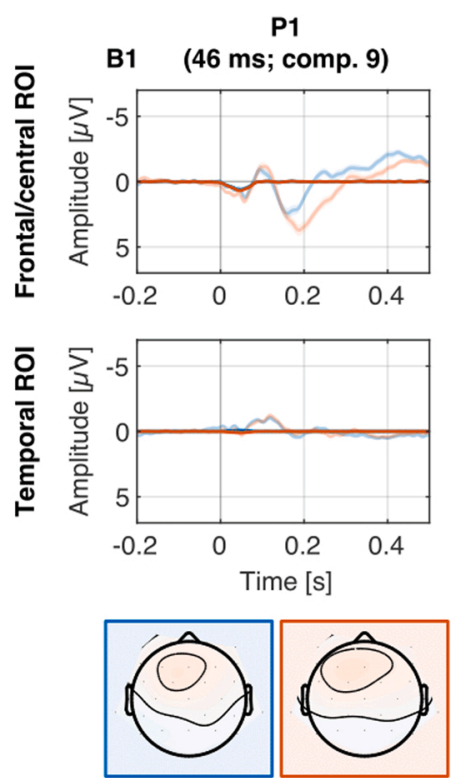

\section{Sound ERPs - Adults}

N1b

B2 (90 ms; comp. 5)
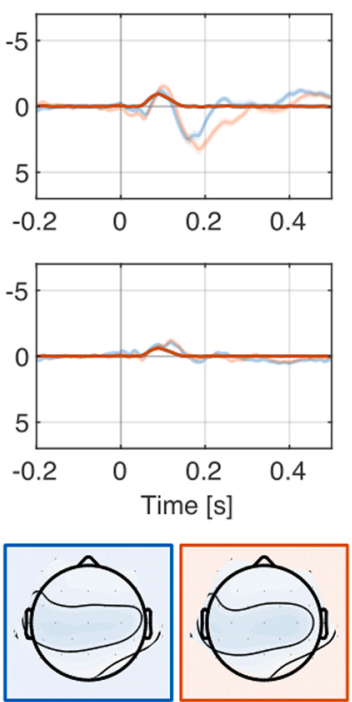

N1c
B3 $\quad(128 \mathrm{~ms} ;$ comp. 7$)$
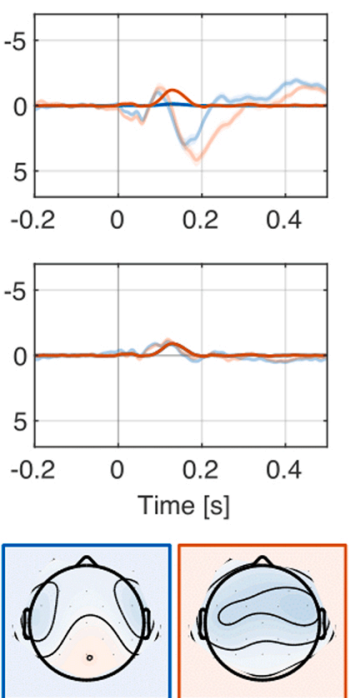

P2

B4 (164 ms; comp. 1)
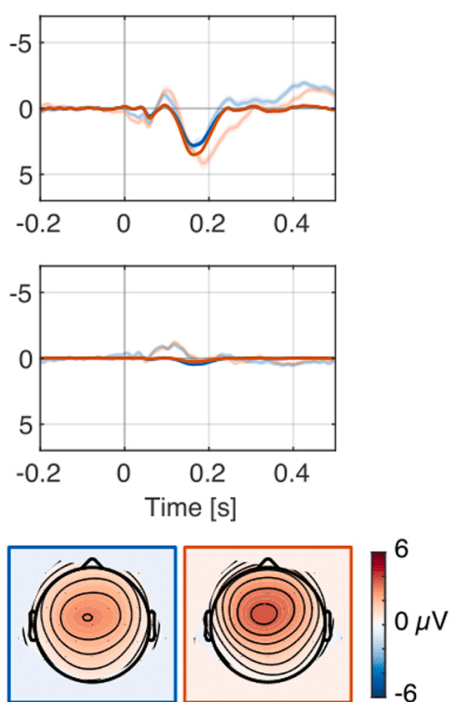

Fig. 3. Reconstructed PCA component difference waveforms (opaque) and grand-average difference waves plus 95\% CIs (transparent) for single (S-M; blue) and random (R-M; red) sound conditions in children and adults. Panel A: Sound evoked components of interest in children. Three positive components were observed (A1, A2, A4) at frontal (Frontal P1: F3, Fz, F4), centro-lateral (Centro-lateral P1: FC5, FC1, FC2, FC6), or fronto-central ROIs (N1c/P2: Cz, FC1, FC2) and are shown in the top row. Frontal P1 and P2 showed negative potentials over temporal ROIs (T7, T8; second row). N1c and P2 components were presumably conflated in the PCA solution in children due to temporal overlap. Bottom row shows component topographies at peak latencies. Panel B: Sound evoked components of interest in adults. Typical P1, N1b, N1c, and P2 sound components were observed. Components (B1-B4) are shown for frontal (P1: F3, F4, Fz, FC1, FC2), central (N1b: C3, C4, Cz), or fronto-central ROIs (N1c/P2: Cz, FC1, FC2) in top row and temporal ROI (T7, T8) in second row. Bottom row shows component topographies at peak latencies. 
Table 2

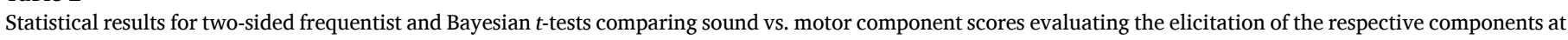

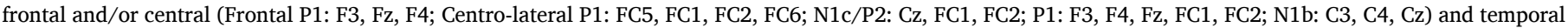
ROIs (electrodes T7 and T8). Significant effects are printed bold.

\begin{tabular}{|c|c|c|c|c|c|c|c|c|}
\hline & \multicolumn{4}{|c|}{ Frontal/central ROIs } & \multicolumn{4}{|c|}{ Temporal ROI } \\
\hline & $d$ & $B F_{10}$ & $t(30)$ & $p$ & $d$ & $B F_{10}$ & $t(30)$ & $p$ \\
\hline \multicolumn{9}{|l|}{ Children } \\
\hline Frontal P1 & 0.85 & 496.68 & 4.74 & $<.001$ & -0.84 & 435.69 & -4.69 & $<.001$ \\
\hline Centro-lateral P1 & 1.23 & $1.09 \times 10^{5}$ & 6.83 & $<.001$ & 0.76 & 141.05 & 4.24 & $<.001$ \\
\hline $\mathrm{N} 1 \mathrm{c} / \mathrm{P} 2$ & 1.66 & $3.80 \times 10^{7}$ & 9.24 & $<.001$ & -1.01 & 4854.06 & -5.62 & $<.001$ \\
\hline \multicolumn{9}{|l|}{ Adults } \\
\hline P1 & 0.64 & 28.18 & 3.58 & .001 & 0.02 & 0.19 & 0.10 & .918 \\
\hline $\mathrm{N} 1 \mathrm{~b}$ & -0.64 & 27.99 & -3.58 & .001 & -0.93 & 1700.11 & -5.22 & $<.001$ \\
\hline $\mathrm{N} 1 \mathrm{c}$ & -0.59 & 14.06 & -3.28 & .003 & -1.39 & $1.10 \times 10^{6}$ & -7.75 & $<.001$ \\
\hline $\mathrm{P} 2$ & 1.50 & $4.66 \times 10^{6}$ & 8.34 & $<.001$ & 0.38 & 1.35 & 2.12 & .042 \\
\hline
\end{tabular}

See Table 2 for statistics.

\section{Discussion}

The current study aimed to establish an understanding of how prediction related psychophysiological processes develop in the context of action-effect couplings. We developed a child-friendly version of a motor-auditory omission paradigm and recorded an electrophysiological omission response in 6-8-year-old children and an adult control group. Participants repeatedly pressed a button triggering a sound which was rarely omitted while watching a silent video. ERP responses were measured in two conditions: a single sound condition where always the same sound was triggered-allowing specific predictions about the identity of the expected sound-and a random sound condition where a random sound was triggered-only allowing unspecific sound predictions. Results show similar omission responses in both age groups. The earliest observed omission response was larger in children than in adults and larger in response to identity-specific predictions compared to identity-unspecific predictions. The pattern of sound-related brain responses in the same time range notably differed between children and adults.

In the following we will discuss (1) the omission response and its subcomponents in children and adults (2) the dissociation of specific and unspecific predictions (3) early oN1 amplitude differences between groups (4) the role of the task-related attentional focus, (5) the development of sound-related brain responses in the N1 range and (6) the role of motor related activity.

\subsection{Omission-related brain responses}

In line with previous studies in adults using versions of the motorsound omission paradigm, an oN1 in response to unexpected omission of sounds was observed in both age groups (Fig. 2). The oN1 is thought to reflect a cortical sensory prediction error resulting from the comparison between predictions, provided by higher cortical levels, and actual sensory input (Dercksen et al., 2020; SanMiguel et al., 2013a, 2013b; van Laarhoven et al., 2017). Specifically, important higher cortical levels involved in motor-sensory prediction seem to be the (supplementary) motor area (Jo et al., 2019; Lima et al., 2016; Pazen et al., 2020; Reznik et al., 2015; Schneider and Mooney, 2018) and the cerebellum (Baumann et al., 2015; Kilteni and Ehrsson, 2020; Knolle et al., 2013; Pazen et al., 2020). These areas, either through direct cortical (motor cortex) or indirect subcortical (cerebellum) connections, influence activity in the auditory cortex, presumably based on a predicted model of the planned movement. The observed results suggest that the above-described pathways of motor-sensory prediction are functional in 6-8-year-old children.

PCA extracted two components in the time range of the oN1 over temporal electrode sites in both age groups (Fig. 2). The early oN1 peaked at $90 \mathrm{~ms}$ in adults and $130 \mathrm{~ms}$ in children and the late oN1 peaked at $148 \mathrm{~ms}$ in adults and $180 \mathrm{~ms}$ in children. In Dercksen et al. (2020) only a single oN1 component was extracted. However, the late oN1 and strong oN2 (which is elicited in a similar time-window but at frontal electrodes) might have been conflated in this study because of their close temporal proximity. In the absence of an oN2, the temporal early and late oN1 omission components have been observed by Korka et al. (2020), who used two buttons and only two different tones for the unpredictable condition (in our study termed random condition). In the current study, the oN2 was also absent or severely reduced because of diverted attention. Furthermore, the use of component loss rotation (Scharf and Nestler, 2019) possibly aided in revealing the late oN1, as it is substantially less prone to conflating components with strong temporal and spatial overlap than other rotation methods.

\subsection{Dissociation of specific and unspecific predictions}

The present study dissociates psychophysiological mechanisms underlying specific (single condition) and unspecific (random condition) auditory predictions. Very similar patterns between age groups were observed regarding the differences between these conditions. The amplitude of the early oN1 was larger in the single sound compared to the random sound condition in both age groups, while the late oN1 had similar amplitudes between conditions in both age groups (Fig. 2). The higher amplitude early oN1 in the single condition compared to the random condition is in line with the findings of SanMiguel et al. (2013a) and Dercksen et al. (2020). This was discussed to reflect specific and unspecific predictions along the sound processing hierarchy, where precision weighting might influence the strength of prediction error (Dercksen et al., 2020). The observed similar responses between age groups suggests that-like adults-children can implement predictions flexibly, both using specific predictions resulting in prediction errors that are attributed a high weight, and more general predictions resulting in prediction errors that are attributed a low weight. These findings contribute to the idea that prediction errors play an important role in learning processes. Learning and sensory prediction error are closely connected in models of motor control (Shadmehr et al., 2010; Imamizu, 2010) and speech production (Hickok and Poeppel, 2007; Pickering and Garrod, 2013). In these models, sensory prediction errors calibrate action in the face of various sources of noise by adapting the internal model that produces both the action and the sensory representation of its consequences. The current study contributes to these ideas by revealing a neuroscientific demonstration of such action-effect prediction errors, arguably in its purest form (using omission), to be present in children. Additionally, we find these prediction error responses to be relatively mature (as compared with sound processing responses) and sophisticated as they distinguish between specific and unspecific violations. Such findings fit well to theoretical accounts that consider prediction (Emberson, 2017; Gredebäck et al., 2018; Köster et al., 2020; Stahl and Feigenson, 2015, 2017; Trainor, 2012) as well as action (Copete et al., 2016; Hunnius and Bekkering, 2014; Koziol et al., 2012; Koziol and Lutz, 
2013) not to be consequences, but rather drivers of cognitive development.

\subsection{Early oN1 amplitude difference between groups}

In contrast to the similar effects between conditions, a larger absolute amplitude of the early oN1 was observed in children compared to adults. One explanation for this could be that children had to allocate more attention to the experimental task of pressing the button in a fixed rhythm. Behavioural results indicate that the child group had more difficulties compared to adults, pressing the button too soon/early more often. Increased attention or mental effort devoted to the task, and therefore to sounds vs. the movie possibly increased the early oN1 response, similar to effects of task-related attention on the sound evoked N1 (Lange, 2013). On the other hand, children were perhaps more drawn to the movie as it was suited for a young age group, making it difficult to determine the exact effect of attention in this paradigm. However, participants were constantly monitored by the experimenter, who controlled that at least overt attention was focused on the screen. An alternative explanation of the higher oN1 amplitudes in children is that many ERP amplitudes are generally higher in children, and decrease into adulthood possibly due to, e.g., a decrease in grey matter (synaptic pruning; Itier and Taylor, 2004; Segalowitz et al., 2010). Lastly, a number of studies in adults have hypothesised a shift in weighting from sensory input to the predictive model as age increases (Moran et al., 2014; Chan et al., 2017; Wolpe et al., 2016). This shift is thought to be influenced by the deteriorating sensory precision because of aging, but also by an increased precision of the predictive model as a consequence of experience. Given the latter, the higher amplitude omission responses in children could be explained as a consequence of a still imprecise predictive model, where high weight is assigned to sensory input in order to update the model. A similar interpretation of the oN1 has recently been proposed by van Laarhoven et al. (2020) in the context of autism, where people suffering from autism demonstrated to assign a uniform, inflexibly high weight to prediction errors (Van de Cruys et al., 2014). However, whether this hypothesis can be transferred to sensory predictions in children has to be addressed in future studies.

\subsection{Attentional focus}

An interesting consequence of the adapted experimental paradigm is the potential effect of attentional focus on the elicitation of prediction error. Several changes had to be implemented to make the experiment suitable for children compared to the study of Dercksen et al. (2020). For example, the experiment had fewer trials, a slightly higher proportion of omissions, and lower sound volume. However, the most notable change was the addition of a silent video during the experiment, which diverted attention to the visual modality. Given that the other changes were small (omission ratio $15 \%$ instead of $12 \%, 10.1 \mathrm{~dB}$ lower sound volume as compared to Dercksen et al., 2020), we assume that this diverted attention was the main driver of the observed differences between the current study and similar omission studies with adults. Compared to similar studies (e.g., Dercksen et al., 2020; Stekelenburg and Vroomen, 2015; Van Laarhoven et al., 2017) the oN1 amplitude was slightly reduced (although no statistical tests were performed). A diminished response would be in line with the conclusions of Chennu et al. (2016): although not specifically focused on the oN1, they used dynamic causal modelling to infer that attention modulates the strength and precision of downward predictions (and thus, theoretically, of the resulting omission response). The current study adds to these findings by demonstrating that directed attention is not required to elicit the oN1, but that diverted attention might result in a diminished amplitude of the component. The dampening effect as a presumed consequence of diverted attention was even more pronounced in the subsequent ERP components that normally follow the oN1 as observed in previous studies (e.g., oN2 and oP3; Dercksen et al., 2020; SanMiguel et al., 2013a).

\subsection{Age differences in sound-related brain responses}

Of special interest was the presumed relation of the sound-related N1 with the oN1 (SanMiguel et al., 2013b). It has been assumed that the generation of predictions induces a pattern of activity that involves shared sources and similar time courses like those of the predicted stimulus (Bastos et al., 2012; SanMiguel et al., 2013b). The current study indicates a largely developed function of cortical sensory prediction error underlying the oN1 component in children aged 6-8 years. In contrast, sound evoked N1 subcomponents in children were either absent or significantly different from adults. Instead of an N1b, children ERPs were dominated by a large amplitude, triple-peaked positive component between $\sim 80$ and $170 \mathrm{~ms}$, followed by a negative peak N2 between $\sim 200$ and $250 \mathrm{~ms}$, which is typical for the age group (Bruneau and Gomot, 1998; Čeponien et al., 1998, 2002; Ponton et al., 2000; Silva et al., 2017; Wunderlich and Cone-Wesson, 2006). The three extracted subcomponents of the positivity around $80-170 \mathrm{~ms}$ in children presumably reflect a frontal P1 at $80 \mathrm{~ms}$, a centro-lateral P1 at $114 \mathrm{~ms}$, and a P2 at $162 \mathrm{~ms}$ (Fig. 3). In contrast, adults showed the standard P1-N1-P2-N2 morphology at central electrodes.

The frontal distribution of the P1 component-that is associated with stimulus encoding processes (Liegeois-Chauvel et al., 1994)-and their observed latencies in the respective age groups are in line with existing literature (Wunderlich and Cone-Wesson, 2006). It has been discussed that the polarity inversion at temporal electrodes, that we also observed in the present study, could partly reflect the Na of the T-complex, even if a different developmental time course of $\mathrm{P} 1$ and $\mathrm{Na}$ in early childhood suggests some independence of the sources underlying P1 and $\mathrm{Na}$ (Shafer et al., 2015). While a bifurcation of the P1 component over fronto-central leads in children has been interpreted as the first sign of N1b (vertex N1) development in previous studies (Gilley et al., 2005; Sussman et al., 2008), PCA did not extract a N1b component. This suggests an absence of the N1b in children, which is in line with the protracted development of the N1b observed in other children studies, particularly when relatively short interstimulus intervals (lower than around $1 \mathrm{~s}$ ) were used (Čeponien et al., 1998; Eggermont and Ponton, 2003; Wetzel and Schröger, 2007). The lack of the vertex N1 and the existence of the oN1 in children indicates a clear dissociation of mechanisms underlying both components. Based on visual inspection of the topographies displayed in Fig. 3, it could be speculated that the centro-lateral P1 extracted by PCA (Fig. 3) resembles the topography of the adult N1b with inverted polarity. Whether both components reflect partly overlapping mechanisms of sound processing might be an interesting question that can be addressed in further developmental studies.

In adults, the vertex $\mathrm{N} 1$ was temporally followed by another subcomponent of the $\mathrm{N} 1$ family, the bilateral temporal N1c (Fig. 3 panel B3). The N1c emerges in early childhood at temporal leads with increased amplitudes and latencies compared to adults. In 4-8-year-olds the N1c peaks $170 \mathrm{~ms}$ after stimulus onset in response to sounds (Bruneau et al., 1997). In the current study, the N1c was probably conflated with the P2 due to strong temporal overlap, peaking around $160 \mathrm{~ms}$ (Fig. 3 panel A4). The prominent P2 component can be observed early in childhood, can be reliably identified in the auditory ERP of children, and most studies report a similar latency and topography in primary school age children and adults (Wunderlich and Cone-Wesson, 2006). In contrast to children, adults do not show a polarity inversion at temporal leads in the time range of the P2 component (Fig. 3, panels A1/A4 \& B1/B4). This supports the assumption that the temporal negative pattern in the $\mathrm{P} 2$ range reflects the N1c in children. The topography of the sound evoked N1c of both groups with a maximum over temporal leads resembles the topography of the oN1, which might indicate at least partly similar sources of activation in the auditory cortex. The finding that both the N1c and the oN1 are strongly elicited in children can be considered additional evidence to support such a link. Taken together, a largely matured response to the unexpected omission of an expected sound is elicited in both age groups while sound-related brain responses show 
significant developmental differences, demonstrating that the prediction error differs from obligatory sound processing.

\subsection{The role of motor related activity}

An assumption of the current paradigm is that by subtracting the motor control condition from the single and random conditions, these latter conditions only reflect sound and omission related brain activity. This way, potential differences in motor activity between adults and children in this study should not influence the conclusions regarding sound and omission responses. Given the typical sound responses obtained in both age groups after motor subtraction, the assumption that single and random conditions only reflect sound and omission related activity seems valid. Nevertheless, little is known about how the interaction between motor and sensory activity develops as the brain matures, especially in the framework of prediction. Motor activity related to voluntary movement has been shown to differ in comparison with adults in terms of latency, amplitude, polarity and oscillatory power, where large differences have been observed until at least 10 years of age (Cheyne et al., 2014; Huo et al., 2011; Johnson et al., 2019; Trevarrow et al., 2019). How such differences influence the interaction between motor and sensory activity is an important subject that could be addressed in future studies.

\section{Conclusion}

Both an early and late oN1 component were observed in children and adults. Children's basic oN1 morphologies and topographies were similar to adults, which is especially interesting given the pronounced differences in sound-related ERPs between groups. Children also show sophisticated processing of identity-specific and identity-unspecific stimuli, demonstrating that they implement specific, higher-weight predictions and more general, lower-weight predictions as efficient as adults. The mature manifestation of action-effect omission responses in children can be considered psychophysiological evidence supporting the important role ascribed to prediction and action as drivers of cognitive development. Furthermore, PCA revealed three distinct subcomponents of the positive peak in the auditory ERP of children, an absence of the $\mathrm{N} 1 \mathrm{~b}$, and a presence of the temporal N1c. The early maturation of both the oN1 and the N1c, as well as similar topographies, suggests similar sources. Apart from the developmental perspective, the study shows elicitation of the oN1 in the absence of directed attention, making omission a suitable tool to study prediction in children and patients. However, absence of attention did seem to have a dampening effect on the component amplitudes, especially the components following the oN1. Finally, omission results seem congruent with earlier omission findings that apply motor-auditory couplings.

\section{Funding}

This work was supported by the Center for Behavioral Brain Sciences Magdeburg financed by the European Regional Development Fund (ZS/ 2016/04/78120) and Leibniz Association (P58/2017).

\section{CRediT authorship contribution statement}

Tjerk T. Dercksen: Conceptualization, Methodology, Software, Validation, Formal analysis, Investigation, Data curation, Writing original draft, Writing - review \& editing. Andreas Widmann: Conceptualization, Methodology, Software, Validation, Formal analysis, Data curation, Writing - review \& editing, Visualization. Florian Scharf: Methodology, Writing - review \& editing. Nicole Wetzel: Conceptualization, Methodology, Resources, Writing - review \& editing, Supervision, Project administration, Funding acquisition.

\section{Declaration of Competing Interest}

None.

\section{Data/code availability}

The dataset generated for this study is available on request to the corresponding author.

\section{Acknowledgements}

We thank all families and parents who enabled the conduction of this study. We thank Dunja Kunke, Gabriele Schöps, Celine Jakel, Lisa Pitschmann and Luisa Kocherscheid for support in data acquisition.

\section{References}

Adolph, K.E., Hoch, J.E., 2019. Motor development: embodied, embedded, enculturated, and enabling. Annu. Rev. Psychol. 70, 141-164.

Albrecht, R., Suchodoletz, W.V., Uwer, R., 2000. The development of auditory evoked dipole source activity from childhood to adulthood. Clin. Neurophysiol. 111 (12), 2268-2276.

Arnal, L.H., Giraud, A.L., 2012. Cortical oscillations and sensory predictions. Trends Cogn. Sci. 16 (7), 390-398.

Barry, R.J., De Blasio, F.M., Fogarty, J.S., Karamacoska, D., 2016. ERP Go/NoGo condition effects are better detected with separate PCAs. Int. J. Psychophysiol. 106, 50-64.

Bakeman, R., 2005. Recommended effect size statistics for repeated measures designs. Behav. Res. Methods 37 (3), 379-384.

Basirat, A., Dehaene, S., Dehaene-Lambertz, G., 2014. A hierarchy of cortical responses to sequence violations in three-month-old infants. Cognition 132 (2), 137-150.

Bastos, A.M., Usrey, W.M., Adams, R.A., Mangun, G.R., Fries, P., Friston, K.J., 2012. Canonical microcircuits for predictive coding. Neuron 76 (4), 695-711.

Baumann, O., Borra, R.J., Bower, J.M., Cullen, K.E., Habas, C., Ivry, R.B., Paulin, M.G., et al., 2015. Consensus paper: the role of the cerebellum in perceptual processes. Cerebellum 14 (2), 197-220.

Bendixen, A., SanMiguel, I., Schröger, E., 2012. Early electrophysiological indicators for predictive processing in audition: a review. Int. J. Psychophysiol. 83 (2), 120-131.

Bigdely-Shamlo, N., Mullen, T., Kothe, C., Su, K.M., Robbins, K.A., 2015. The PREP pipeline: standardized preprocessing for large-scale EEG analysis. Front. Neuroinform. 9, 16.

Boldin, A.M., Geiger, R., Emberson, L.L., 2018. The emergence of top-down, sensory prediction during learning in infancy: a comparison of full-term and preterm infants. Dev. Psychobiol. 60 (5), 544-556.

Bonmassar, C., Widmann, A., Wetzel, N., 2020. The impact of novelty and emotion on attention-related neuronal and pupil responses in children. Dev. Cogn. Neurosci. 42, 100766.

Brainard, D.H., 1997. The psychophysics toolbox. Spat. Vis. 10 (4), 433-436.

Bruneau, N., Gomot, M., 1998. Auditory evoked potentials (N1 wave) as indices of cortical development. In: Neuroimaging in Child Neuropsychiatric Disorders. Springer, Berlin, Heidelberg, pp. 113-123.

Bruneau, N., Roux, S., Guerin, P., Barthelemy, C., Lelord, G., 1997. Temporal prominence of auditory evoked potentials (N1 wave) in 4-8-year-old children. Psychophysiology 34 (1), 32-38.

Burton, M., Starzak, R. , 2015. Shaun the Sheep Movie [Film]. Aardman Animations.

Čeponien, R., Cheour, M., Näätänen, R., 1998. Interstimulus interval and auditory eventrelated potentials in children: evidence for multiple generators. Electroencephalogr. Clin. Neurophysiol./Evoked Potentials Sect. 108 (4), 345-354.

Čeponien, R., Rinne, T., Näätänen, R., 2002. Maturation of cortical sound processing as indexed by event-related potentials. Clin. Neurophysiol. 113 (6), 870-882.

Chan, J.S., Wibral, M., Wollstadt, P., Stawowsky, C., Brandl, M., Helbling, S., Kaiser, J., et al., 2017. Predictive coding over the lifespan: increased reliance on perceptual priors in older adults-a magnetoencephalography and dynamic causal modelling study. bioRxiv, 178095

Chennu, S., Noreika, V., Gueorguiev, D., Shtyrov, Y., Bekinschtein, T.A., Henson, R., 2016. Silent expectations: dynamic causal modeling of cortical prediction and attention to sounds that weren't. J. Neurosci. 36 (32), 8305-8316.

Cheyne, D., Jobst, C., Tesan, G., Crain, S., Johnson, B., 2014. Movement-related neuromagnetic fields in preschool age children. Hum. Brain Mapp. 35 (9), $4858-4875$.

Copete, J.L., Nagai, Y., Asada, M., 2016. Motor development facilitates the prediction of others' actions through sensorimotor predictive learning. In: Proceedings of the 2016 Joint IEEE International Conference on Development and Learning and Epigenetic Robotics (ICDL-EpiRob). IEEE, pp. 223-9.

Delorme, A., Makeig, S., 2004. EEGLAB: an open source toolbox for analysis of singletrial EEG dynamics including independent component analysis. J. Neurosci. Methods 134 (1), 9-21.

Dercksen, T.T., Widmann, A., Schröger, E., Wetzel, N., 2020. Omission related brain responses reflect specific and unspecific action-effect couplings. NeuroImage 215, 116840. 
Dien, J., 2012. Applying principal components analysis to event-related potentials: a tutorial. Dev. Neuropsychol. 37 (6), 497-517.

Dien, J., 1998. Addressing misallocation of variance in principal components analysis of event-related potentials. Brain Topogr. 11 (1), 43-55.

Eggermont, J.J., Ponton, C.W., 2003. Auditory-evoked potential studies of cortical maturation in normal hearing and implanted children: correlations with changes in structure and speech perception. Acta Oto-laryngol. 123 (2), 249-252.

Elsner, B., 2007. Infants' imitation of goal-directed actions: the role of movements and action effects. Acta Psychol. 124 (1), 44-59.

Emberson, L.L., 2017. How does experience shape early development? Considering the role of top-down mechanisms. In: Advances in Child Development and Behavior, 52 JAI, pp. 1-41.

Emberson, L.L., Richards, J.E., Aslin, R.N., 2015. Top-down modulation in the infant brain: learning-induced expectations rapidly affect the sensory cortex at 6 months. Proc. Natl. Acad. Sci. USA 112 (31), 9585-9590.

Feldman, H., Friston, K., 2010. Attention, uncertainty, and free-energy. Front. Hum. Neurosci. 4, 215.

Friston, K., 2005. A theory of cortical responses. Philos. Trans. R. Soci. B: Biol. Sci. 360 (1456), 815-836.

Gilley, P.M., Sharma, A., Dorman, M., Martin, K., 2005. Developmental changes in refractoriness of the cortical auditory evoked potential. Clin. Neurophysiol. 116 (3), 648-657.

Gredebäck, G., Lindskog, M., Juvrud, J.C., Green, D., Marciszko, C., 2018. Action prediction allows hypothesis testing via internal forward models at 6 months of age. Front. Psychol. 9, 290.

Hickok, G., Poeppel, D., 2007. The cortical organization of speech processing. Nat. Revi. Neurosc. 8 (5), 393-402.

Hunnius, S., Bekkering, H., 2014. What are you doing? How active and observational experience shape infants' action understanding. Philos. Trans. R. Soc. B: Biol. Sci. 369 (1644), 20130490.

Huo, X., Wang, Y., Kotecha, R., Kirtman, E.G., Fujiwara, H., Hemasilpin, N., Xiang, J., et al., 2011. High gamma oscillations of sensorimotor cortex during unilateral movement in the developing brain: a MEG study. Brain Topogr. 23 (4), 375-384.

Imamizu, H., 2010. Prediction of sensorimotor feedback from the efference copy of motor commands: a review of behavioral and functional neuroimaging studies. Jpn. Psychol. Res. 52 (2), 107-120.

Itier, R.J., Taylor, M.J., 2004. Effects of repetition and configural changes on the development of face recognition processes. Dev. Sci. 7 (4), 469-487.

Jennrich, R.I., 2004a. Derivative free gradient projection algorithms for rotation. Psychometrika 69 (3), 475-480.

Jennrich, R.I., 2004b. Rotation to simple loadings using component loss functions: the orthogonal case. Psychometrika 69 (2), 257-273.

Jennrich, R.I., 2006. Rotation to simple loadings using component loss functions: the oblique case. Psychometrika 71 (1), 173-191.

Jo, H.G., Habel, U., Schmidt, S., 2019. Role of the supplementary motor area in auditory sensory attenuation. Brain Struct. Funct. 224 (7), 2577-2586.

Johnson, B., Jobst, C., Al-Loos, R., He, W., Cheyne, D., 2019. Developmental changes in movement related brain activity in early childhood. BioRxiv, 531905.

Joos, K., Gilles, A., Van de Heyning, P., De Ridder, D., Vanneste, S., 2014. From sensation to percept: the neural signature of auditory event-related potentials. Neurosci. Biobehav. Rev. 42, 148-156.

Kilteni, K., Ehrsson, H.H., 2020. Functional connectivity between the cerebellum and somatosensory areas implements the attenuation of self-generated touch. J. Neurosci. 40 (4), 894-906.

Klug, M., Gramann, K., 2020. Identifying key factors for improving ICA-based decomposition of EEG data in mobile and stationary experiments. bioRxiv.

Knolle, F., Schröger, E., Baess, P., Kotz, S.A., 2012. The cerebellum generates motor-toauditory predictions: ERP lesion evidence. J. Cogn. Neurosci. 24 (3), 698-706.

Knolle, F., Schröger, E., Kotz, S.A., 2013. Cerebellar contribution to the prediction of selfinitiated sounds. Cortex 49 (9), 2449-2461.

Korka, B., Schröger, E., Widmann, A., 2020. What exactly is missing here? The sensory processing of unpredictable omissions is modulated by the specificity of expected action-effects. Eur. J. Neurosci. 52 (12), 4667-4683.

Köster, M., Kayhan, E., Langeloh, M., Hoehl, S., 2020. Making sense of the world: infant learning from a predictive processing perspective. Perspect. Psychol. Sci. 15 (3), 562-571.

Koziol, L.F., Budding, D.E., Chidekel, D., 2012. From movement to thought: executive function, embodied cognition, and the cerebellum. Cerebellum 11 (2), 505-525.

Koziol, L.F., Lutz, J.T., 2013. From movement to thought: the development of executive function. Appl. Neuropsychol.: Child 2 (2), 104-115.

Lange, K., 2013. The ups and downs of temporal orienting: a review of auditory temporal orienting studies and a model associating the heterogeneous findings on the auditory N1 with opposite effects of attention and prediction. Front. Hum. Neurosci. 7, 263.

Lee, M.D., Wagenmakers, E.J., 2013. Bayesian data analysis for cognitive science: a practical course.

Liegeois-Chauvel, C., Musolino, A., Badier, J.M., Marquis, P., Chauvel, P., 1994. Evoked potentials recorded from the auditory cortex in man: evaluation and topography of the middle latency components. Electroencephalogr. Clin. Neurophysiol./Evoked Potentials Sect. 92 (3), 204-214.

Lima, C.F., Krishnan, S., Scott, S.K., 2016. Roles of supplementary motor areas in auditory processing and auditory imagery. Trends Neurosci. 39 (8), 527-542.

Mahajan, Y., McArthur, G., 2013. Maturation of the auditory t-complex brain response across adolescence. Int. J. Dev. Neurosci. 31 (1), 1-10.

Moran, R.J., Symmonds, M., Dolan, R.J., Friston, K.J., 2014. The brain ages optimally to model its environment: evidence from sensory learning over the adult lifespan. PLoS Comput. Biol. 10 (1), e1003422.
R.D. Morey, J.N. Rouder BayseFactor: Computation of Bayes Factors for Common Designs. R Package Version 0.9.12-14.2 (2018) https://CRAN.R-project.org/package =BayesFactor.

Näätänen, R., Picton, T., 1987. The N1 wave of the human electric and magnetic response to sound: a review and an analysis of the component structure. Psychophysiology 24 (4), 375-425.

Oldfield, R.C., 1971. The assessment and analysis of handedness: the Edinburgh inventory. Neuropsychologia 9 (1), 97-113.

Paulus, M., Hunnius, S., Van Elk, M., Bekkering, H., 2012. How learning to shake a rattle affects 8-month-old infants' perception of the rattle's sound: electrophysiological evidence for action-effect binding in infancy. Dev. Cogn. Neurosci. 2 (1), 90-96.

Pazen, M., Uhlmann, L., van Kemenade, B.M., Steinsträter, O., Straube, B., Kircher, T., 2020. Predictive perception of self-generated movements: commonalities and differences in the neural processing of tool and hand actions. NeuroImage 206, 116309.

Pickering, M.J., Garrod, S., 2013. An integrated theory of language production and comprehension. Behav. Brain Sci. 36 (04), 329-347.

Picton, T.W., Alain, C., Woods, D.L., John, M.S., Scherg, M., Valdes-Sosa, P., Trujillo, N. J., et al., 1999. Intracerebral sources of human auditory-evoked potentials. Audiol. Neurotol. 4 (2), 64-79.

Pion-Tonachini, L., Kreutz-Delgado, K., Makeig, S., 2019. ICLabel: an automated electroencephalographic independent component classifier, dataset, and website. NeuroImage 198, 181-197.

Ponton, C., Eggermont, J.J., Khosla, D., Kwong, B., Don, M., 2002. Maturation of human central auditory system activity: separating auditory evoked potentials by dipole source modeling. Clin. Neurophysiol. 113 (3), 407-420.

Ponton, C.W., Eggermont, J.J., Kwong, B., Don, M., 2000. Maturation of human central auditory system activity: evidence from multi-channel evoked potentials. Clin. Neurophysiol. 111 (2), 220-236.

R Core Team, 2014. R: A Language and Environment for Statistical Computing. R Foundation for Statistical Computing, Vienna, Austria. 〈http://www.R-project.org/〉.

Reznik, D., Ossmy, O., Mukamel, R., 2015. Enhanced auditory evoked activity to selfgenerated sounds is mediated by primary and supplementary motor cortices. J. Neurosci. 35 (5), 2173-2180.

Rinker, T., Shafer, V.L., Kiefer, M., Vidal, N., Yu, Y.H., 2017. T-complex measures in bilingual Spanish-English and Turkish-German children and monolingual peers. PLoS One 12 (3), e0171992.

SanMiguel, I., Saupe, K., Schröger, E., 2013a. I know what is missing here: electrophysiological prediction error signals elicited by omissions of predicted "what" but not "when". Front. Hum. Neurosci. 7, 407.

SanMiguel, I., Widmann, A., Bendixen, A., Trujillo-Barreto, N., Schröger, E., 2013b. Hearing silences: human auditory processing relies on preactivation of soundspecific brain activity patterns. J. Neurosci. 33 (20), 8633-8639.

Scharf, F., Nestler, S., 2019. A comparison of simple structure rotation criteria in temporal exploratory factor analysis for event-related potential data. Methodology.

Schneider, D.M., Mooney, R., 2018. How movement modulates hearing. Annu. Rev. Neurosci. 41, 553-572.

Schröger, E., Marzecová, A., SanMiguel, I., 2015. Attention and prediction in human audition: a lesson from cognitive psychophysiology. Eur. J. Neurosci. 41 (5), 641-664.

Segalowitz, S.J., Santesso, D.L., Jetha, M.K., 2010. Electrophysiological changes during adolescence: a review. Brain Cogn. 72 (1), 86-100.

Shadmehr, R., Smith, M.A., Krakauer, J.W., 2010. Error correction, sensory prediction, and adaptation in motor control. Annu. Rev. Neurosci. 33, 89-108.

Shafer, V.L., Yan, H.Y., Wagner, M., 2015. Maturation of cortical auditory evoked potentials (CAEPs) to speech recorded from frontocentral and temporal sites: three months to eight years of age. Int. J. Psychophysiol. 95 (2), 77-93.

Shin, Y.K., Proctor, R.W., Capaldi, E.J., 2010. A review of contemporary ideomotor theory. Psychol. Bull. 136 (6), 943-974.

Silva, L.A.F., Magliaro, F.C.L., Carvalho, A.C.M., Matas, C.G., 2017. Maturation of long latency auditory evoked potentials in hearing children: systematic review. CoDAS 29 (No. 3), e20160107.

Stahl, A.E., Feigenson, L., 2015. Observing the unexpected enhances infants' learning and exploration. Science 348 (6230), 91-94.

Stahl, A.E., Feigenson, L., 2017. Expectancy violations promote learning in young children. Cognition 163, 1-14.

Stekelenburg, J.J., Vroomen, J., 2015. Predictive coding of visual-auditory and motor auditory events: an electrophysiological study. Brain Res. 1626, 88-96.

Sussman, E., Steinschneider, M., Gumenyuk, V., Grushko, J., Lawson, K., 2008. The maturation of human evoked brain potentials to sounds presented at different stimulus rates. Hear. Res. 236 (1-2), 61-79.

Tonnquist-Uhlen, I., Ponton, C.W., Eggermont, J.J., Kwong, B., Don, M., 2003. Maturation of human central auditory system activity: the T-complex. Clin. Neurophysiol. 114 (4), 685-701.

Trainor, L.J., 2012. Predictive information processing is a fundamental learning mechanism present in early development: evidence from infants. Int. J. Psychophysiol. 83 (2), 256-258.

Trevarrow, M.P., Kurz, M.J., McDermott, T.J., Wiesman, A.I., Mills, M.S., Wang, Y.P., Wilson, T.W., et al., 2019. The developmental trajectory of sensorimotor cortical oscillations. Neuroimage 184, 455-461.

Van de Cruys, S., Evers, K., Van der Hallen, R., Van Eylen, L., Boets, B., de-Wit, L., Wagemans, J., 2014. Precise minds in uncertain worlds: predictive coding in autism. Psychol. Rev. 121 (4), 649-675.

van Laarhoven, T., Stekelenburg, J.J., Eussen, M.L., Vroomen, J., 2020. Atypical visualauditory predictive coding in autism spectrum disorder: electrophysiological evidence from stimulus omissions. Autism 24 (7), 1849-1859. 
van Laarhoven, T., Stekelenburg, J.J., Vroomen, J., 2017. Temporal and identity prediction in visual-auditory events: electrophysiological evidence from stimulus omissions. Brain Res. 1661, 79-87.

Wetzel, N., Schröger, E., 2007. Modulation of involuntary attention by the duration of novel and pitch deviant sounds in children and adolescents. Biol. Psychol. 75 (1), 24-31.

Wetzel, N., Widmann, A., Schröger, E., 2011. Processing of novel identifiability and duration in children and adults. Biol. Psychol. 86 (1), 39-49.

Winkler, I., Debener, S., Müller, K.R., Tangermann, M., 2015. On the influence of highpass filtering on ICA-based artifact reduction in EEG-ERP. In: Proceedings of the 2015 37th Annual International Conference of the IEEE Engineering in Medicine and Biology Society (EMBC). IEEE, pp. 4101-5.

Winkler, I., Háden, G.P., Ladinig, O., Sziller, I., Honing, H., 2009. Newborn infants detect the beat in music. Proc. Natl. Acad. Sci. USA 106 (7), 2468-2471.
Wunderlich, J.L., Cone-Wesson, B.K., 2006. Maturation of CAEP in infants and children: a review. Hear. Res. 212 (1-2), 212-223.

Wolpaw, J.R., Penry, J.K., 1975. A temporal component of the auditory evoked response. Electroencephalogr. Clin. Neurophysiol. 39 (6), 609-620.

Wolpe, N., Ingram, J.N., Tsvetanov, K.A., Geerligs, L., Kievit, R.A., Henson, R.N., Rowe, J.B., et al., 2016. Ageing increases reliance on sensorimotor prediction through structural and functional differences in frontostriatal circuits. Nat. Commun. 7 (1), 1-11.

Woods, D.L., 1995. The component structure of the N 1 wave of the human auditory evoked potential. Electroencephalogr. Clin. Neurophysiol.-Suppl. 44, 102-109.

Zhang, F., Jaffe-Dax, S., Wilson, R.C., Emberson, L.L., 2019. Prediction in infants and adults: a pupillometry study. Dev. Sci. 22 (4), e12780. 